University of Nebraska - Lincoln

DigitalCommons@University of Nebraska - Lincoln

\title{
Model complexity affects transient population dynamics following a dispersal event: A case study with pea aphids
}

Brigitte Tenhumberg

University of Nebraska - Lincoln, btenhumberg2@unl.edu

Andrew J. Tyre

University of Nebraska-Lincoln, atyre2@unl.edu

Richard Rebarber

University of Nebraska - Lincoln, rrebarber1@unl.edu

Follow this and additional works at: https://digitalcommons.unl.edu/bioscifacpub

Part of the Life Sciences Commons

Tenhumberg, Brigitte; Tyre, Andrew J.; and Rebarber, Richard, "Model complexity affects transient population dynamics following a dispersal event: A case study with pea aphids" (2009). Faculty Publications in the Biological Sciences. 94.

https://digitalcommons.unl.edu/bioscifacpub/94

This Article is brought to you for free and open access by the Papers in the Biological Sciences at DigitalCommons@University of Nebraska - Lincoln. It has been accepted for inclusion in Faculty Publications in the Biological Sciences by an authorized administrator of DigitalCommons@University of Nebraska - Lincoln. 


\title{
Model complexity affects transient population dynamics following a dispersal event: a case study with pea aphids
}

\author{
Brigitte Tenhumberg, ${ }^{1,4}$ Andrew J. Tyre, ${ }^{2}$ and Richard Rebarber ${ }^{3}$ \\ ${ }^{1}$ School of Biological Sciences and Department of Mathematics, University of Nebraska, Lincoln, Nebraska 68588-0118 USA \\ ${ }^{2}$ School of Natural Resources, University of Nebraska, Lincoln, Nebraska 68583 USA \\ ${ }^{3}$ Department of Mathematics, University of Nebraska, Lincoln, Nebraska 68588 USA
}

\begin{abstract}
Stage-structured population models predict transient population dynamics if the population deviates from the stable stage distribution. Ecologists' interest in transient dynamics is growing because populations regularly deviate from the stable stage distribution, which can lead to transient dynamics that differ significantly from the stable stage dynamics. Because the structure of a population matrix (i.e., the number of life-history stages) can influence the predicted scale of the deviation, we explored the effect of matrix size on predicted transient dynamics and the resulting amplification of population size. First, we experimentally measured the transition rates between the different life-history stages and the adult fecundity and survival of the aphid, Acythosiphon pisum. Second, we used these data to parameterize models with different numbers of stages. Third, we compared model predictions with empirically measured transient population growth following the introduction of a single adult aphid. We find that the models with the largest number of life-history stages predicted the largest transient population growth rates, but in all models there was a considerable discrepancy between predicted and empirically measured transient peaks and a dramatic underestimation of final population sizes. For instance, the mean population size after 20 days was 2394 aphids compared to the highest predicted population size of 531 aphids; the predicted asymptotic growth rate $\left(\lambda_{\max }\right)$ was consistent with the experiments. Possible explanations for this discrepancy are discussed.
\end{abstract}

Key words: Acythosiphon pisum; aphids; life-history stages; matrix population models; population dynamics; transient amplification.

\section{INTRODUCTION}

Structured population models are common tools used to examine population dynamics. Most researchers focus on analyzing the long-term, asymptotic population growth rate $\left(\lambda_{\max }\right.$, the largest eigenvalue of the population projection matrix), the rate at which the population grows at the stable stage distribution (but see Burgman et al. 1993, Koons et al. 2005, 2007, Caswell 2007, Townley et al. 2007). However, a population can be perturbed away from the stable stage distribution by disturbances such as environmental catastrophes, selective harvesting regimes, and management actions (e.g., animal release and translocation programs); even in established populations the assumption that the population growth follows the asymptotic growth is unwarranted in many cases (e.g., Clutton-Brock and Coulson 2002, Koons et al. 2005, Townley et al. 2007). Deviations away from the stable stage distribution change the population dynamics, resulting in sometimes dramatically different transient dynamics (Townley et al. 2007). The scale of the deviation and the time until the population returns to the stable stage distribution

Manuscript received 7 September 2008; accepted 6 October 2008. Corresponding Editor: L. Stone.

${ }^{4}$ E-mail: btenhumberg2@unl.edu depend upon the population matrix structure, parameter values, and hence the net reproductive value of a population (Koons et al. 2005). If matrix size influences predicted transient dynamics it raises the question of how many life-history stages need to be included in a matrix to accurately predict the nature of transient population dynamics of real populations.

Short-term dynamics are highly relevant for many ecological systems (Hastings 2004). Transient dynamics not only influence short-term dynamics but they can significantly alter the long-term population size as well (Hodgson and Townley 2004, Koons et al. 2005). Caswell (2001:95) wrote "More comparisons of asymptotic and transient dynamics would be useful." As a result scientists have started to explore the ecological implications of this phenomenon, called "transient amplification" (Neubert and Caswell 1997). In this paper we define the transient amplification as a ratio of the predicted transient and asymptotic population sizes. The numerator is population size starting from an unstable stage distribution (transient growth), and the denominator is population size starting from the stable stage distribution (asymptotic growth). A transient amplification of 2.5 means that the population that started from a particular unstable stage distribution is 2.5 times larger than a population of the same initial size at the stable stage distribution. Since the stable stage 
distribution is a unique property of a population matrix, the population size after $t$ time steps assuming asymptotic growth is highly predictable $\left(\lambda_{\max }^{t}\right)$. In contrast, there is a different transient dynamic for every possible initial population structure. It has been suggested that one can take advantage of transient behavior in population management (Koons et al. 2005) by manipulating the stage distribution to temporarily increase the population growth rate (and thus reduce the extinction risk). D. Hodgson, R. Rebarber, and S. Townley (unpublished data) explore how to exploit transient dynamics in captive breeding programs.

Although it is common to estimate the parameters of projection matrices by analyzing life-history events for individuals, as far as we know the predictions of matrix models parameterized in this way have rarely been empirically tested. The logistical difficulties in observing populations for many years in relatively constant environments are enormous. Bierzychudek (1999) constructed matrix models for two Arisaema triphyllum populations and measured population sizes 15 years later; only one of the populations performed as predicted. This could be attributable to poor parameter estimation due to limited data or to changing environmental conditions and consequently changing parameter values. Pfister and Stevens (2003) evaluated the accuracy of matrix models as mean field approximation for individual-based models. They argued that matrix models do not always capture long-term population growth trajectories in the presence of large individual variation in growth; similar reservations might hold for predicted transient dynamics.

We estimated stage-structured matrix models for the aphid Acyrthosiphon pisum from data on individual lifehistory events, explored the effect of model complexity on predicted transient dynamics, and compared predicted transients with empirically observed transients. We also compared our observed population growth rates with those predicted by another model that exhibits strong transient dynamics (Gross et al. 2002); the parameters of this model were estimated from stagestructured time series data. Our laboratory experiments confirm that real populations can show transient amplification. The models with the highest number of stage classes are most consistent with the experimental results, although all models show ecologically significant departures in total population size from observed.

\section{Material And Methods}

Life history of Acyrthosiphon pisum

Pea aphids, Acyrthosiphon pisum (Harris) (Homoptera: Aphididae), feed on the phloem of alfalfa plants and other legumes (Dixon 1985; see Plate 1). From spring to autumn aphids are viviparous (give birth to live young) and parthenogenic (asexual reproduction); under laboratory conditions the production of sexual morphs can be completely avoided. Aphid populations consist of winged (alatae) and unwinged (apterae) morphs; if feeding conditions become unfavorable (e.g., low host plant quality or crowded conditions) an increased proportion of winged morphs are born, which disperse to new habitats (Dixon 1985). Wingless morphs usually remain in the vicinity of their birth location unless they are disturbed (e.g., by predators; Nelson and Rosenheim 2006, Nelson 2007), thus single plants may host a few generations of aphids. Aphids have five distinct lifehistory stages: four juvenile instars and one adult stage (Hutchinson and Hogg 1984, 1985), a fact that makes them ideal candidates for stage-structured models. Acyrthosiphon pisum have a very short generation time and can develop from first instar to reproducing adult in only 10 days (Campbell and Mackauer 1977). This rapid development makes it feasible to observe aphid populations for several generations, which is a prerequisite for experimentally studying transient population dynamics.

\section{Experiment 1: Estimating model parameters}

We conducted this experiment in an insect rearing room under controlled conditions $\left(23-25^{\circ} \mathrm{C}, 12 \mathrm{~h}\right.$ daylight, 40 $50 \%$ relative humidity). We transferred single wingless adult aphids to clip cages (4 $\mathrm{cm}$ diameter) and attached them to three-week-old alfalfa plants (Medicago sativa L., cv. 'Vernal'; one cage per plant). Each pot $(12.5 \mathrm{~cm}$ diameter) contained four to six alfalfa plants, and each pot contained up to four clip cages. After 12 hours we removed all aphids, except one first-instar aphid per cage, which were then followed through their life cycle development. We began with 80 aphids and discarded two cages in which adults did not produce any offspring; this left us with a sample size of 78 aphids. Each cage was identified by a unique number, allowing the identification of each individual aphid. We recorded aphid development and mortality in daily intervals. Exuviae in the cages indicated transitions to the next developmental stage and were removed upon discovery. In 11 cages we overlooked an exuvia; these aphids were only used to determine adult fecundity. Thus, we calculated the stage-specific frequency of daily transitions to the next developmental stage based on 67 aphids (see the matrix model for calculation of transition probabilities), and the fecundity was recorded from all 63 aphids that survived to the reproductive stage. Once aphids molted to the adult stage we counted and removed all newborns daily until all adult aphids died. Adult survival and fecundity was analyzed using the statistical software R (R Development Core Team 2006). We modeled daily offspring production as a Poisson process and used parametric survival analysis to estimate age-independent (exponentially distributed survival times) and age-dependent (Weibull distribution) survival functions. We also compared these parametric survival curves to nonparametric Kaplan Meier survival estimates (Cox and Oakes 1984).

\section{Experiment 2:}

\section{Measuring transient population dynamics (time series)}

We used the same insect rearing rooms as in the previous experiment, except the temperature increased 
TABLE 1. Matrix models of the pea aphid Acyrthosiphon pisum.

\begin{tabular}{lllll}
\hline \hline \multicolumn{1}{c}{ Model } & \multicolumn{1}{c}{$\mathbf{N}_{1}$} & \multicolumn{1}{c}{$\lambda_{\max }$} & $N_{20}$ & $T_{20}$ \\
\hline Two juvenile stages + one adult stage & $(0,0,1)$ & $1.29(1.26,1.31)$ & $293(216,415)$ & $2.49(2.37,2.66)$ \\
Two juvenile stages + two adult stages & $(0,0,0,1)$ & $1.23(1.21,1.26)$ & $143(106,201)$ & $2.64(2.52,2.85)$ \\
Four juvenile stages + one adult stage & $(0,0,0,0,1)$ & $1.25(1.23,1.27)$ & $170(131,228)$ & $2.43(2.32,2.60)$ \\
Four juvenile stages + two adult stages & $(0,0,0,0,0,1)$ & $1.21(1.19,1.23)$ & $100(77,133)$ & $2.60(2.47,2.81)$ \\
Two juvenile stages + seven adult stages & $(0,0,0,1,0,0,0,0,0)$ & $1.30(1.28,1.33)$ & $529(370,784)$ & $3.53(3.36,3.8)$ \\
Four juvenile stages + seven adult stages & $(0,0,0,0,0,1,0,0,0,0,0)$ & $1.27(1.24,1.29)$ & $298(222,414)$ & $3.42(3.25,3.69)$ \\
Seven juvenile pseudo-stages & $(0,0,0,0,0,0,0,0,1,0,0,0,0,0)$ & $1.27(1.25,1.32)$ & $283(229,518)$ & $3.05(2.75,3.68)$ \\
$\quad+$ seven adult stagest & & & &
\end{tabular}

Notes: Transient amplification after 20 days is calculated as $N_{20} /\left(\lambda_{\max }\right)^{19}$, where $N_{20}=\Sigma_{i} n_{i, 20}$ and $n_{i, 20}$ indicates the number of aphids in stage class $i, 20$ days after releasing the founder aphids, which we assume to be in the second adult age class. The numbers in parentheses are upper and lower 95\% confidence intervals using bootstrapping.

$\uparrow$ During bootstrapping the means and variances of juvenile developmental time and consequently the number of pseudo-stages changed. Thus, the 95\% confidence intervals are based on matrices with 6-9 juvenile pseudo-stages.

by $2^{\circ} \mathrm{C}$ unintentionally as a result of fluctuation in the air-conditioning system of the building. We transferred one adult aphid of unknown age to a large cage $(45 \mathrm{~cm}$ wide $\times 55 \mathrm{~cm}$ high $\times 50 \mathrm{~cm}$ deep) that initially contained a single three-week-old alfalfa plant; we used a sample size of 12 cages. Because our aphid cultures contained very few winged (alate) aphids, we used wingless adults to start this experiment. Compared to wingless (apterous) aphids, alate $A$. pisum have a slightly lower total fecundity $\left(10 \%\right.$ at $19.7^{\circ} \mathrm{C}$; Campbell and Mackauer 1977); some authors report lower fecundity of alate aphids during the first few days of adulthood (the gross reproductive rate was $20 \%$ lower in a field population in Wisconsin, USA; Hutchinson and Hogg 1984). We do not know of any study reporting fitness differences between offspring of alate and apterous aphids.

For the next 20 days we counted aphid numbers in daily intervals. To prevent density dependence from affecting aphid population dynamics we increased the number of plants in each cage to a maximum of eight plants per cage. We did not follow the population dynamics for a longer time because with increasing aphid abundance it is increasingly difficult to accurately count aphids and to avoid density-dependent effects on aphid survival and fecundity. Additionally, based on our model predictions, population growth rates reached an asymptote after 20 days.

\section{Matrix model}

Our models consider population dynamics in the absence of density dependence, such as in the early phase of colonizing a new habitat (e.g., an alfalfa field). We constructed stage-structured birth flow models with a time step of one day. The models varied in the number of juvenile and adult stages. To account for agedependent fecundity and mortality (Table 1), our most complex models divided adults into seven different stages: the first adult stage represents pre-reproductive adults; the next five stages have durations of $d=3 \mathrm{~d}$ and the last one lumps all remaining ages into a single age class (Appendix A). The last adult age class had a very small contribution to population growth as a result of very low fecundity. Then we successively reduced the model structure to a $3 \times 3$ matrix model (first and second instar; third and fourth instar; adults), as used by Gross et al. (2002). All models had the same general form:

$$
\left(\begin{array}{ccccc}
a_{11} & 0 & \ldots & f_{s-1} & f_{s} \\
a_{21} & a_{22} & \ldots & 0 & 0 \\
0 & a_{32} & \ddots & \vdots & \vdots \\
\vdots & \vdots & \ddots & a_{s-1, s-1} & 0 \\
0 & 0 & \ldots & a_{s, s-1} & a_{s s}
\end{array}\right)
$$

where $s$ is the total number of stages in the model, the $a_{i, j}$ are transition probabilities, and the $f_{i}$ are the fertilities or contributions to the youngest stage class by reproducing adults.

The transitions $a_{i, j}$ indicate the rate of surviving individuals graduating from stage $j$ to $i$. For larval instars we could directly estimate these rates from observed transitions using

$$
\hat{a}_{i j}=\frac{m_{i j}}{\sum_{h} m_{h j}}
$$

where $m_{i, j}$ is the number of individuals in stage $i$ that were in stage $j$ the previous time step. Because all models considering two to four juvenile stages underestimated observed transient peaks, we also constructed a model with a negative binomial distribution of juvenile ages within stages, which generally increases transient dynamics (Caswell 2001). This model delays individuals in their progress through each larval instar by dividing each juvenile period into $k$ pseudo-stages. If $T$ is the mean duration and $V\left(T_{i}\right)$ the variance in duration of a larval instar, then the number of pseudo-stages, $k_{i}$, and their transition probabilities, $\gamma_{i}$, are calculated as follows:

$$
\begin{aligned}
k_{i} & =\frac{\bar{T}_{i}^{2}}{V\left(T_{i}\right)+\bar{T}_{i}} \\
\gamma_{i} & =\frac{\bar{T}_{i}}{V\left(T_{i}\right)+\bar{T}_{i}} .
\end{aligned}
$$

Assuming the pre-reproductive survival is $\sigma_{i}$, then the transition to the next juvenile stage $a_{i+1, j}$ is $\sigma_{i} \gamma_{i}^{k_{i}}$. For 
adults, the $a_{i, j}$ are the products of the graduation and survival probabilities. The probability of graduating to the next stage for an adult is $1 / d$ where $d$ is the duration of the stage, assuming a geometric distribution of individuals within stages. For the stage-specific survival of adults we use geometric averages over the entire stage. Survival was modeled as a Weibull distribution; hence the death rate at age $x$ (McCallum 2000, Tenhumberg et al. $2004 a$ ) is

$$
\kappa \rho(\rho x)^{\kappa-1}
$$

where $\rho$ is the rate, and $\kappa$ is the shape parameter. Hence the probability to survive from age $x$ to $x+d$ is

$$
\bar{\mu}_{x \text { to } x+d}=\exp \left[-\int_{x}^{x+d} \kappa \rho(\rho u)^{\kappa-1} d u\right]=e^{(\rho x)^{\kappa}-[\rho(x+d)]^{\kappa}}
$$

and the per time step survival of adults in stage $i$ is $P_{i}=$ $\left(\bar{\mu}_{x \text { to } x+d}\right)^{1 / d}$.

The daily reproductive output of an adult aphid, the fecundity $b_{i}$, was modeled using a smooth spline term in a generalized additive model with a log link and Poisson error distribution.

The type of parameterization can affect the magnitude of transients; Caswell and Neubert (2005) found that postbreeding census birth pulse models have larger transients than pre-breeding birth pulse models. However, aphids reproduce more or less continuously during adulthood fecundity so $a_{1, j}$ is best approximated with a birth flow model; the fertility of individuals in stage $i, f_{i}$, is

$$
f_{i}=\sqrt{P_{1}}\left(\frac{b_{i}+\sum_{j} t_{j i} b_{j}}{2}\right)
$$

where $P_{1}$ is the survival rate of first-instar A. pisum, $b_{i}$ is the fecundity of adults in stage $i$, and $t_{j i}$ accounts for individuals that are transitioning from stage $i$ to $j$ (Caswell 2001). This formula takes into account the change in birth rate of individuals transitioning to the next stage within a single population census interval; it also assumes that on average all observed first-instar larvae have survived half of a census interval.

We projected the population growth rate, $N_{t+1} / N_{t}$, following from the introduction of a single adult aphid. If the model consisted of more than one reproductive age class we assumed that the aphid belonged to the second youngest reproductive age class. It is safe to assume that our source population (our main aphid culture) was at the stable stage distribution, which is highly skewed toward younger adults (data not shown).

\section{Transient amplification}

Let $\mathbf{N}_{1}$ be the initial population vector, and $\mathbf{M}_{1}$ be the stable stage distribution vector of the same total population size as $\mathbf{N}_{1}$. Here we use $T_{t, N_{1}}$ to denote the transient amplification at time $t$ with initial population $\mathbf{N}_{1}$, which is the ratio of population size at $t$ starting from initial state $\mathbf{N}_{1}$ (hence under the influence of transient dynamics) to the population size that would have existed at $t$ assuming the population started with $\mathbf{M}_{1}$ and grew at the asymptotic population growth rate. Thus, $T_{t, N_{1}}=1$ means the population is the same size at $t$ whether it started from $\mathbf{M}_{1}$ (asymptotic growth) or from some other stage distribution $\mathbf{N}_{1}$ (transient growth). Transient amplification depends on the initial stage distribution $\left(\mathbf{N}_{1}\right)$; different initial stage distributions lead to greater or lesser degrees of amplification. Throughout the paper we use a single initial stage vector with one aphid in the first reproductive class, so we simplify the notation and use $T_{t}$ instead of $T_{t, N_{1}}$. Transient amplification is therefore defined as

$$
T_{t}=\left(\sum_{i} n_{i, t}\right) /\left(\lambda_{\max }\right)^{t-1}
$$

where $n_{i, t}$ indicates the number of aphids in stage class $i$ on day $t$. For example, $T_{20}=2$ indicates that 20 days after the arrival of the first individual (i.e., one adult aphid) population size is twice as high as expected under asymptotic growth with normalized initial data. Assuming asymptotic growth, the expected population size at time $t$ is proportional to $\left(\lambda_{\max }\right)^{t-1}$; if we start with a single aphid the expected population size equals $\left(\lambda_{\max }\right)^{t-1}$.

\section{Bootstrapping confidence limits}

We used nonparametric bootstrapping to generate 95\% confidence limits for $\lambda_{\max }, T_{20}$, and the total population size at 20 days, $N_{20}$. Bootstrapping was carried out by sampling with replacement from the population of individuals in experiment 1. Each selected individual's entire development and reproductive history was included as a single unit; this ensured that withinindividual correlations were maintained. For each bootstrap replicate, the survival curves, development rates, and fecundity models were recalculated, the matrices constructed, and the statistics $\lambda_{\max }, T_{20}$, and $N_{20}$ calculated. We used 10000 bootstrap replicates for each matrix structure and calculated bias-corrected $95 \%$ intervals from these distributions (Efron and Tibshirani 1993).

\section{Monte Carlo simulation of demographic stochasticity}

Predicted population size after 20 days was much smaller than the average observed population size. Because demographic stochasticity can influence population growth rates (e.g., Morris and Doak 2002), we used a Monte Carlo simulation to evaluate whether demographic stochasticity could explain any disagreement between experiment and model prediction. For the simulation we used the programming language $\mathrm{R}$ ( $\mathrm{R}$ Development Core Team 2006). We simulated data from matrices with juvenile pseudo-stages (which is most consistent with observations). We simulated 10 replicates from each of 1000 bootstrapped matrices; each run 


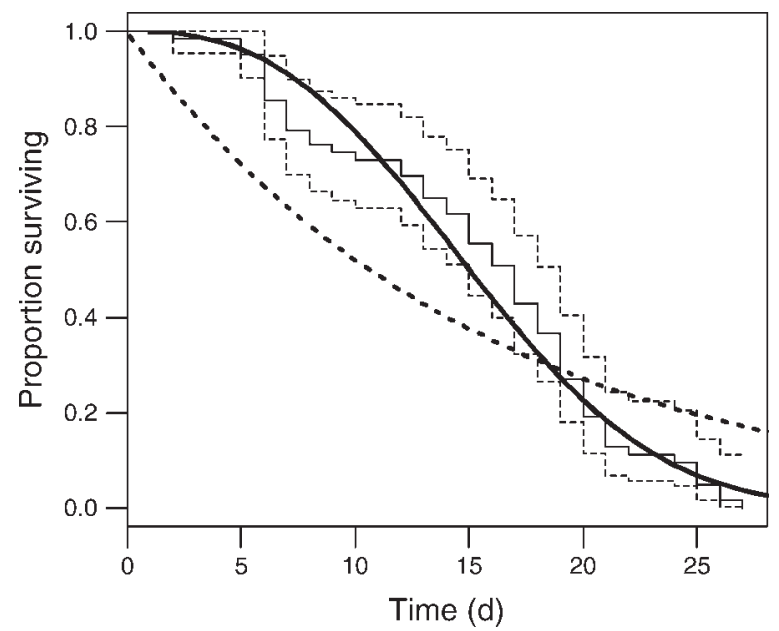

FIG. 1. Survival of reproductive pea aphid (Acyrthosiphon pisum) adults. The thin lines represent mean (solid line) and 95\% confidence limits (dotted lines) of the Kaplan-Meier survival estimates. The thick solid line indicates the survival estimate assuming that survival times follow a Weibull distribution (survival decreased with age, $\rho=0.058025, \kappa=2.660962$, where $\rho$ is the rate, and $\kappa$ is the shape parameter); and the thick dotted line assumes exponentially distributed survival times (age independent).

was initialized with a single aphid in the first reproductive adult class. We then projected the populations forward in time for 20 days, including demographic stochasticity by using Poisson distributions for fecundity and multinomial distributions for survival and growth. At each time step individuals in each stage on a single plant either died, moved up to the next stage, or remained in the current stage according to the probabilities in the matrix; this is a multinomial random variable. For example, if there are 10 second-instar aphids one possible multinomial outcome is two remaining in the first instar, seven growing to the third instar, and one dying. In addition, aphids on a single plant in the $i$ th reproductive stage produced a random number of first-instar aphids from a Poisson distribution with the mean equal to $f_{i} n_{i, t}$; the total number of new first instars is the sum over all reproductive stages. Because we intended to simulate aphid population growth under controlled laboratory conditions, we did not include environmental stochasticity.

\section{Perturbation analysis of the pseudo-stages model}

It is possible that changes in the life-history rates between experiments 1 and 2 are responsible for the discrepancy in the observed and measured transient dynamics. Possible reasons for these changes include small temperature differences during the parameter estimation experiments and the experiment recording transient dynamics, performance differences between single aphids (reared in clip cages) and aphid populations (Allee effects), and maternal effects (high-"quality" founder aphids). We elaborate on these possibilities in the discussion. It is not possible to estimate the matrix parameters from our time series data as Gross et al. (2002) did, because we did not track the stage structure within the growing populations. Doing so would have required destructive sampling and dramatically increased the number of replicates needed. We designed the experiment to get detailed data on the time evolution of total population size. As a result, the matrix parameters are "unidentifiable" based only on the time series of total population size, since there are many parameter combinations that yield similar trajectories.

To get around this issue we used a numerical perturbation analysis to evaluate the magnitude of parameter perturbations required to obtain an approximate match between empirical observations and model predictions. This was similar to the "pattern based modeling" approach (Grimm et al. 2005, Tyre et al. 2007). First, we perturbed vital rates individually to determine which ones influenced transient dynamics the most. For instance, it is reasonable to assume that higher temperature or improved rearing conditions will decrease the mean and variance of larval developmental time. Second, we perturbed combinations of vital rates simultaneously using a grid search and selected the smallest perturbations that matched three different aspects of the empirical results: the timing of the initial transient peak in the growth rate, the amplitude of the initial transient peak, and the total population size on day 20 .

\section{Results}

\section{A. pisum vital rates}

The developmental time for the different juvenile instars were: $1.96 \mathrm{~d}$ (variance $[\mathrm{var}]=0.31$ ) for the first instar, 1.90 $\mathrm{d}(\operatorname{var}=0.27)$ for the second instar, $1.90 \mathrm{~d}(\mathrm{var}=0.63)$ for the third instar, and $2.31 \mathrm{~d}$ (var =0.46) for the fourth instar. After reaching adulthood, aphids went through a pre-reproductive period of $2.03 \mathrm{~d}(\mathrm{var}=0.90)$. The first offspring of the second generation were born eight days after the start of the experiment. The juvenile mortality rate was extremely low; only five of 67 aphids died before reaching adulthood. Adult fecundity and mortality were strongly age dependent. We modeled survival as a Weibull distribution $(\rho=0.058, \kappa=2.66$, Akaike Information Criterion $[\mathrm{AIC}]=414.8$ ); for comparison, modeling survival as an age-independent probability (exponentially distributed survival times) resulted in a very poor fit of our data (Fig. 1; AIC = 471.8). The daily number of offspring produced decreased with age and is consistent with a Poisson process (Fig. 2). We used these results to parameterize seven models by varying the number of juvenile stages (two, four, and pseudo-stages) and the number of adult stages (one, two, and seven; Table 1). Constructing models with more than seven adult stages did not change model predictions (data not shown).

\section{Predicted and observed population dynamics}

We compared three different model predictions with our observed population dynamics: transient growth rates, asymptotic growth rates, and population size. 


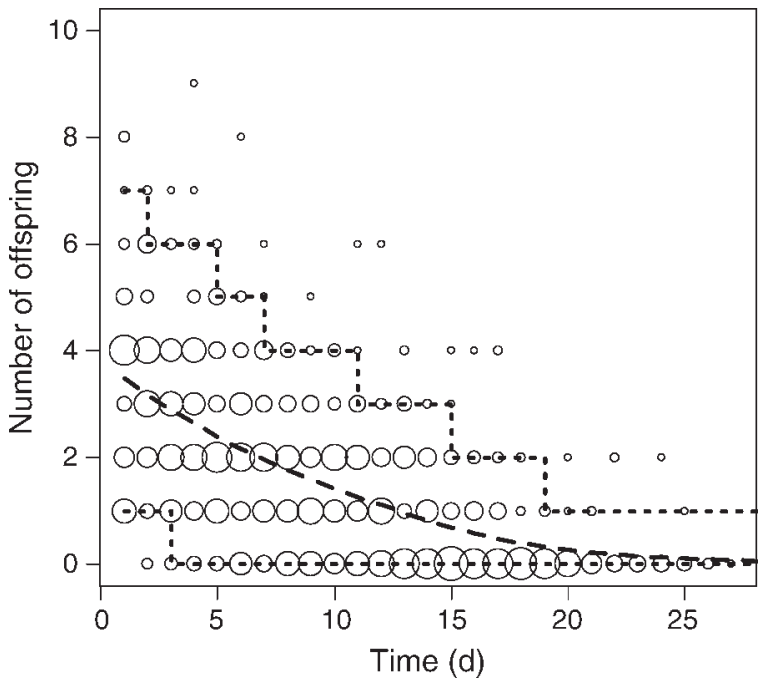

FIG. 2. Fecundity of reproductive Acyrthosiphon pisum adults. The circles indicate the frequency distribution of offspring production for aphids at different ages (area is proportional to frequency). The dashed line is the mean fertility assuming that offspring production follows a Poisson distribution; the dotted lines indicate the 5th and 95th quantile of the Poisson distribution.

Transient growth rates.-Following the release of a single adult aphid the observed population growth rates $\left(N_{t+1} / N_{t}\right)$ clearly oscillated. In all models predicted population growth rates did not change by much during the last three days and reached an asymptote after 20 days (Fig. 3). The observed number of peaks was consistent with model predictions, but the magnitude of the observed peak in growth rates (hereafter "transient peak") was considerably larger than predicted by any of our models.

Model structure had a large influence on the transient population dynamics (Fig. 3). The number of juvenile stages had the largest influence on the predicted transient peaks. The models with only two juvenile stages had no transient peaks, models with four juvenile stages predicted higher transient peaks, and the model including pseudo-stages predicted the largest transient peaks. Among the models with four juvenile stages, the model incorporating the effect of age-dependent fecundity (seven adult stages) predicted the largest transient peaks (Fig. 3). The values reported in Table 1 should be interpreted as upper bounds as other ages of the initial aphid result in smaller transient amplification (Appendix B). Initializing populations with old aphids tends to lead to $T_{20}<1$, which implies that these initial conditions lead to population growth less than asymptotic; these stages account for $<10 \%$ of the adult population at the stable stage distribution.

Asymptotic growth rates.-In our experiments the population growth rate $\left(N_{t+1} / N_{t}\right)$ of the last three days was $1.375 \pm 0.024$ (mean $\pm \mathrm{SE}$ ). The long-term population growth rates (the largest eigenvalue of the

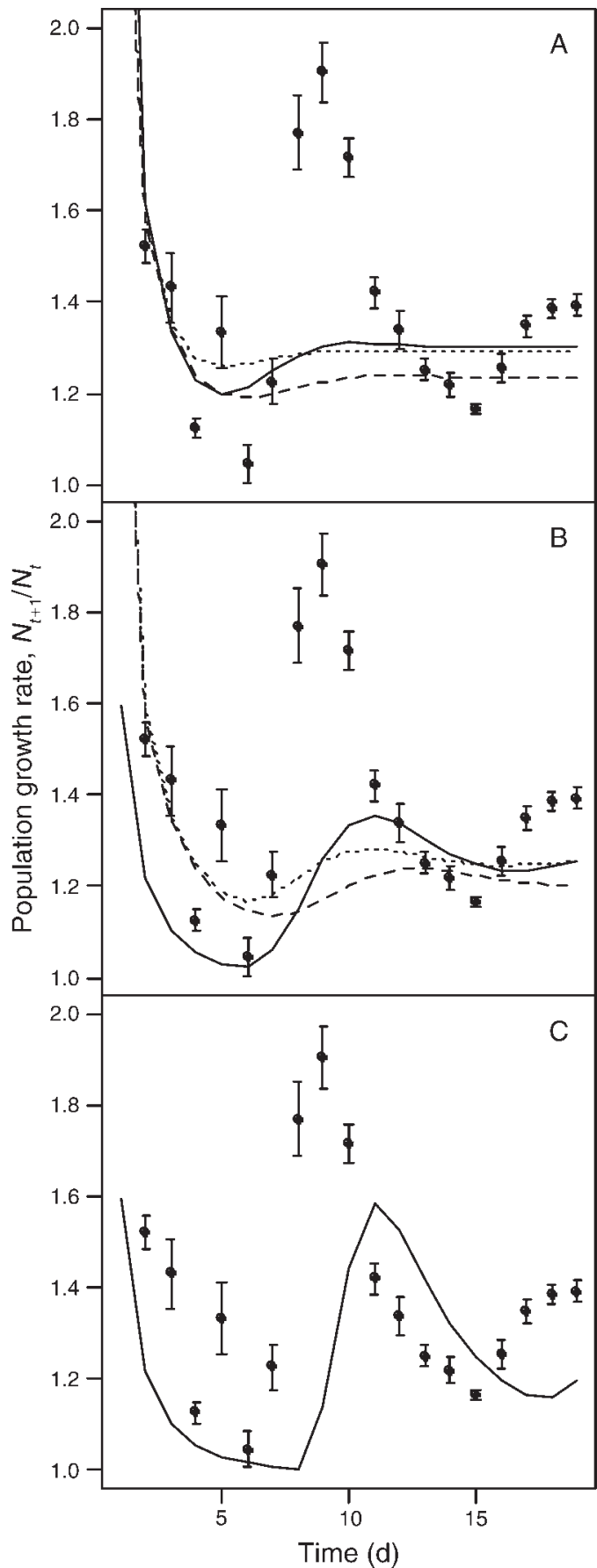

FIG. 3. Predicted transient dynamics as a function of model complexity: (A) two juvenile stages, (B) four juvenile stages, and $(\mathrm{C})$ juvenile pseudostage model (seven pseudostages + seven adult stages). The solid circles indicate empirically measured population dynamics with their $95 \%$ confidence intervals, and the curves indicate predicted population dynamics. Different curves indicate different numbers of adult stages (the solid curve represents seven stages; the dashed curve represents one pre-reproductive and one reproductive stage; the dotted curve represents one adult stage). The initial stage vectors are listed in Table 1. 


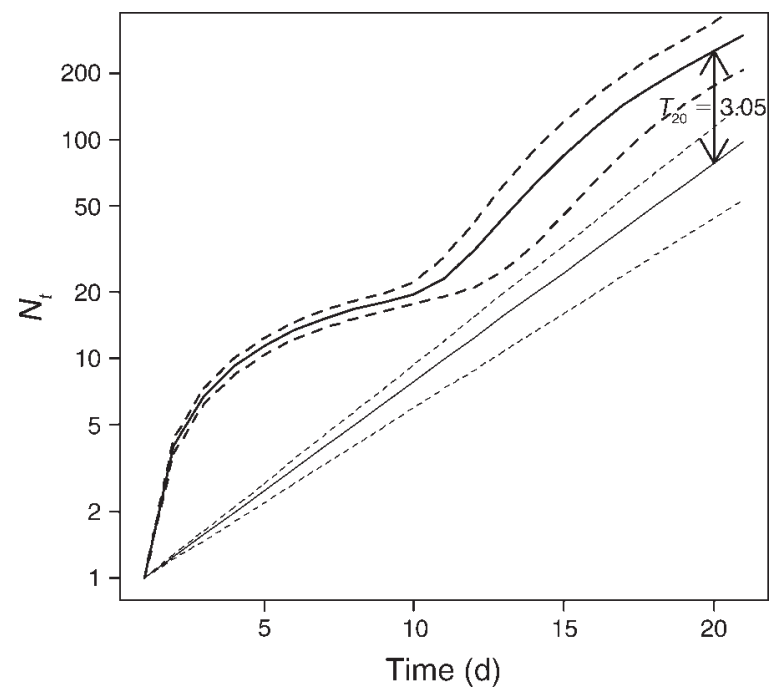

FIG. 4. Mean (solid lines) and 95\% bootstrap confidence limits (dashed lines) for population trajectories under asymptotic (fine lines) and transient amplification (heavy lines) for the pseudo-stages model.

matrix model, $\lambda_{\max }$ ) were similar in all models (confidence limits ranging between 1.19 and 1.33; Table 1), which was slightly lower than 1.375 . This could be due to the somewhat higher temperature $\left(2^{\circ} \mathrm{C}\right)$ in the second experiment; alternatively, the asymptotic growth rate was not yet reached in our experiment.

Predicted and observed population size.-Across all models, population size with transient amplification had nonoverlapping confidence intervals with the corresponding asymptotically growing population (e.g., pseudo-stages model; Fig. 4). Fig. 4 underrepresents the magnitude of the differences; bootstrap replicates with low transient amplification (i.e., the lower confidence limit) also have lower asymptotic growth rates. Nonetheless, the highest confidence limit for population size from any model was 784 (Table 1), still far lower than the empirical observations. During the experiment the number of aphids increased from one aphid $(t=1)$ to a mean of 2394 aphids per cage (1098-3406 aphids, $t=$ $20)$. The model most consistent with the observed shortterm dynamics (largest transient peak, model with pseudo-stages) did not predict the largest transient amplification (Table 1); the largest amplification was predicted from the model with seven adult and two juvenile stages $\left(T_{20}=3.53\right.$; Table 1$)$ although the confidence limits for all models with seven adult stages overlapped broadly. The pseudo-stages model predicted $13 \%$ less transient amplification $\left(T_{20}=3.05\right.$; Table 1$)$.

\section{Monte Carlo simulation of demographic stochasticity}

To evaluate whether the disagreement between predicted and observed population size was due to demographic stochasticity, we simulated a population from the pseudo-stages model using bootstrapped matrices to incorporate parameter uncertainty (Fig. 5).
Some simulation runs had dramatically higher amplifications than the median, but even the extreme values of 10000 simulations were outside the $95 \%$ confidence intervals of the observed population size. Although the lowest extremes of population size were below asymptotic growth, this does not mean that transient amplification is not important or not significantly different from asymptotic growth. The variance of population size grows with time when there is demographic stochasticity (Gotelli 2001). Thus some fraction of runs would always fall below asymptotic growth, even though the mean population size was higher than asymptotic. The distribution of mean growth under transient amplification is always above asymptotic growth (Fig. 4).

\section{Perturbation analysis of the pseudo-stages model}

Decreasing developmental time produced earlier but not higher transient peaks, but shifting adult fecundity to younger ages without increasing the total lifetime reproduction substantially increased transient peaks; decreasing larval survival generally reduced population growth and shifted the growth curves toward smaller values (Fig. 6). In the clip cage experiment almost all larvae survived to adulthood, so it is possible that survival was somewhat lower in the second experiment (large cages). Adult survival had only a minor effect on transient dynamics (Appendix C). Conditions improving aphid performance are likely to affect both developmental time and reproductive output simultaneously, and we obtained the best match to transient population growth rates and change in total population size over time when simultaneously perturbing survival, develop-

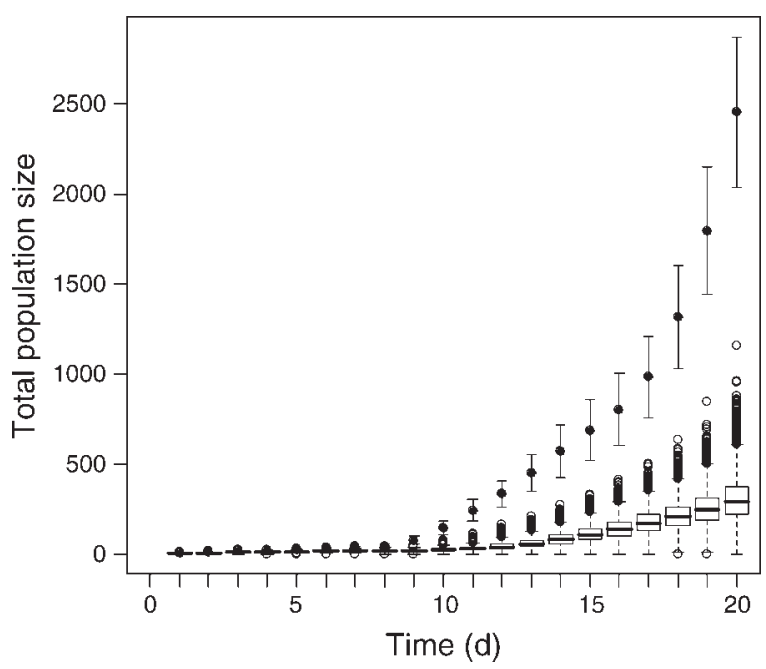

FIG. 5. Comparison of observed and predicted total population sizes of Acyrthosiphon pisum within 20 days after releasing a single adult aphid. The circles are observed means with their $95 \%$ confidence intervals; the box plots represent Monte Carlo simulations based on the model with juvenile pseudo-stages, demographic stochasticity, and parameter uncertainty from bootstrapped matrices. 

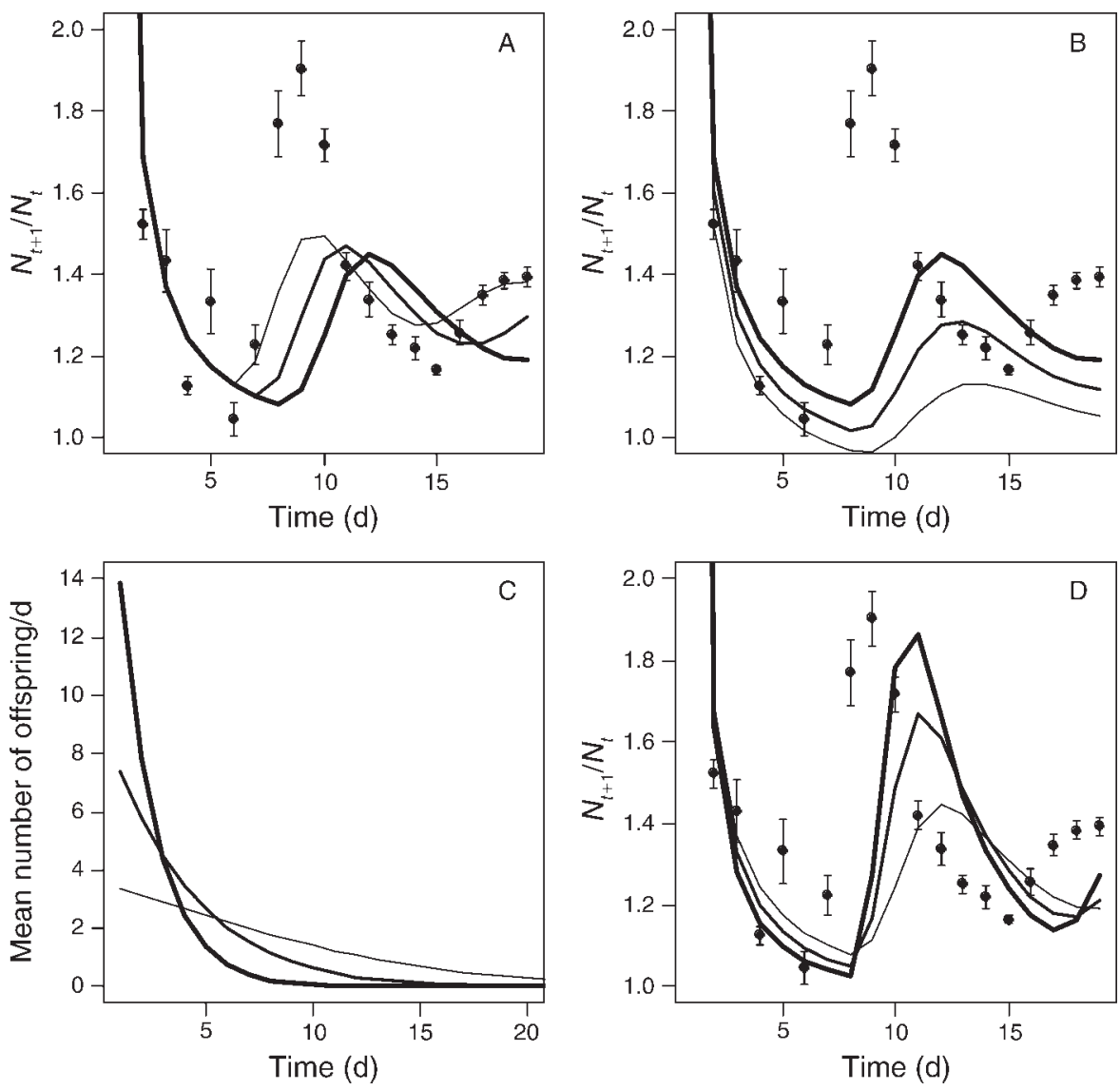

FIG. 6. Effect of perturbations on transient growth rates in the pseudo-stages model. (A) Effect of reducing the mean and variance of larval developmental time by $0 \%, 10 \%$, and $20 \%$; (B) juvenile survival decreased by $0 \%, 10 \%$, and $20 \%$; (C) adult fecundity on day 0 increased by $0 \%, 75 \%$, and $150 \%$; (D) transient growth rates for the corresponding fecundity schedules in panel C. Thinner lines correspond to shorter development time, lower survival, or lower initial fecundity. The circles are empirical observed means with their $95 \%$ confidence intervals.

mental time, and reproduction (Fig. 7). An acceptable match between predicted and measured population dynamics does not require nonlinear dynamics such as an Allee effect.

Compared to the predictions using the unperturbed parameter estimates, using a parameter combination that was most consistent with the observations produced $7 \%$ higher asymptotic population growth rate and a $40 \%$ increase in transient amplification $\left(\lambda_{\max }=1.36[1.29\right.$, 1.43], $T_{20}=4.29$ [3.8, 4.7]; Fig. 7). For all parameter perturbations shown in Fig. 6 we calculated $\lambda_{\max }$ and $T_{20}$; we also included a matrix with fecundity at half the unperturbed value to increase the range. We found that transient amplification increased linearly with $\lambda_{\max }\left(T_{20}\right.$ $=-9.77+10.05 \times \lambda_{\max }, r^{2}=0.78, P<0.001$; Appendix D). In other words, as $\lambda_{\max }$ increases, so does transient amplification. The effect of increasing $\lambda_{\max }$ on population size is already incorporated into $T_{20}$, so any additional increase in $T_{20}$ with increasing $\lambda_{\max }$ indicates a further increase in population size due to transient amplification.
Comparison with the models by Gross et al. (2002)

The model by Gross et al. (2002) used time series data from three Wisconsin A. pisum field populations feeding on alfalfa plants (M. satia cv. 'Vernal') to parameterize models consisting of two juvenile stages (first and second instar and third and fourth instar) and one adult stage, equivalent to our simplest model. The predicted asymptotic population growth rates were within the range of our laboratory populations $\left(\lambda_{\max }=1.18-1.42\right)$. The projected transient dynamics between the three populations have similar oscillation intervals, but differ in the amplitude of the transient peaks and the time until the asymptotic growth rate is reached (transient amplification $=2.65-3.31$; Fig. 8). In general, if transient peaks were large it took longer for the population growth rate to stabilize. Our empirically measured transients had lower frequencies than predicted by Gross et al.'s model, but the predicted amplitudes of the transient peaks were within the range of our observed transient peak. Interestingly, the transient dynamics of Gross et al.'s $3 \times 3$ matrix model were 


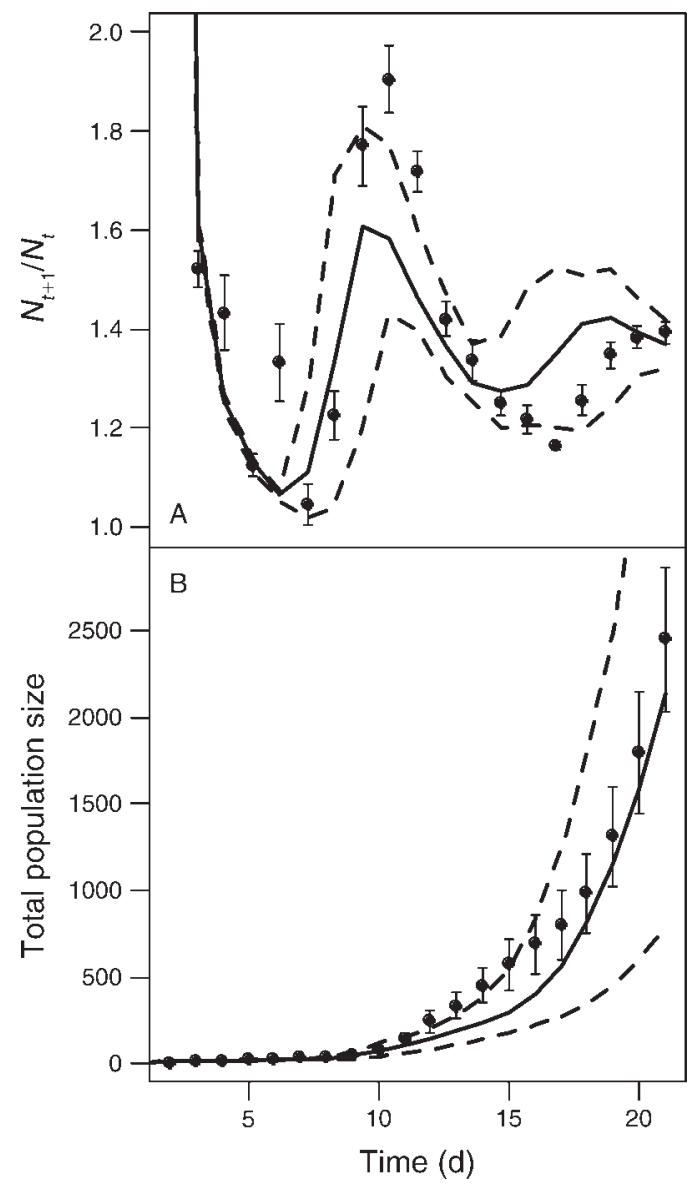

FIG. 7. (A) Comparison of observed and predicted population growth rates and (B) total population sizes of Acyrthosiphon pisum within 20 days after releasing a single adult aphid. The circles are observed means with their 95\% confidence intervals; the solid lines indicate pseudo-stages model prediction under transient population growth, and the dashed lines are bootstrap $95 \%$ confidence intervals. The perturbations for this plot are mean development reduced by $17 \%$, variance in development reduced by $25 \%$, juvenile survival reduced by $6 \%$, and initial fecundity increased by $110 \%$. With these perturbations the median (with bootstrap 95\% confidence limits) asymptotic growth rate, $\lambda_{\max }$, is $1.36(1.29,1.43)$, and the median population growth size 20 days after the arrival of the first individual, $T_{20}$, is $4.29(3.79,4.65)$.

completely different from those predicted by our $3 \times 3$ model for which we measured the transition rates directly.

\section{DisCUSSION}

Most researchers focus on the asymptotic population growth rate that is predicted if the population is at the stable stage distribution. In many cases the assumption of a stable stage distribution is violated during the dispersal phase. Dispersal is often restricted to particular life-history stages, and consequently population growth following a dispersal event may deviate from asymptotic growth. In this paper we mimicked aphid dispersal by using an initial population consisting of a single adult aphid. We demonstrated, both empirically and theoretically, that the population growth rate during the initial dispersal phase of $A$. pisum is larger than the long-term growth rate, $\lambda_{\max }$. These transient amplifications result in considerably higher population densities after 20 days than would be expected under asymptotic growth. This phenomenon is known as population inertia (Koons et al. 2007). During dispersal population densities are low and thus population dynamics are unlikely to be affected by density dependence. In the absence of density dependence, this boost in population density is expected to have long-term effects (Koons et al. 2007). Our results also imply that small variation in temperature has a greater impact on transients than on long-term dynamics, resulting in a striking underestimation of $\lambda_{\max }$-based population growth during the early immigrations phase of aphids. This might be a critical factor in understanding the success of natural enemies in controlling prey population dynamics. We have shown elsewhere that aphid density when the first predators arrive is a key factor determining the probability of a pest outbreak (Tenhumberg 2004). Moreover, Fagan et al. (2005) illustrated the existence of threshold population levels beyond which herbivores were unable to reverse a plant invasion.

Dispersal to new locations is risky because small populations are inherently in danger of extinction as a result of demographic stochasticity (e.g., Tenhumberg et al. 2004b, Drake 2005) and Allee effects (e.g., Keitt et al. 2001, Allen et al. 2005, Taylor and Hastings 2005). A low probability of establishment when population size is small is consistent with the low success rate in establishing biocontrol agents (Grevstad 1999, Stiling 1990, Shea and Possingham 2000, Berggren 2001). High

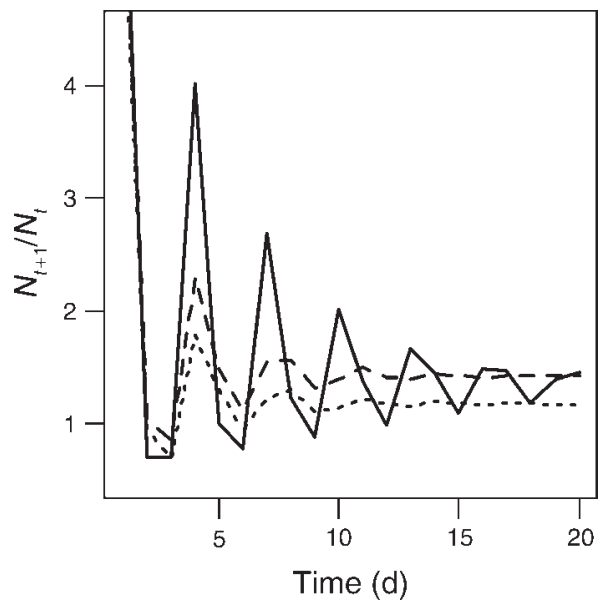

FIG. 8. Predicted transients from the model by Gross et al. (2002) using the initial population vector, $\mathbf{v}=(0,0,1)$. The different curves indicate models for three Wisconsin aphid populations: the solid curve represents Arlington 1980, the dashed curve respresents Madison 1982, and the dotted curve represents Arlington 1982. 


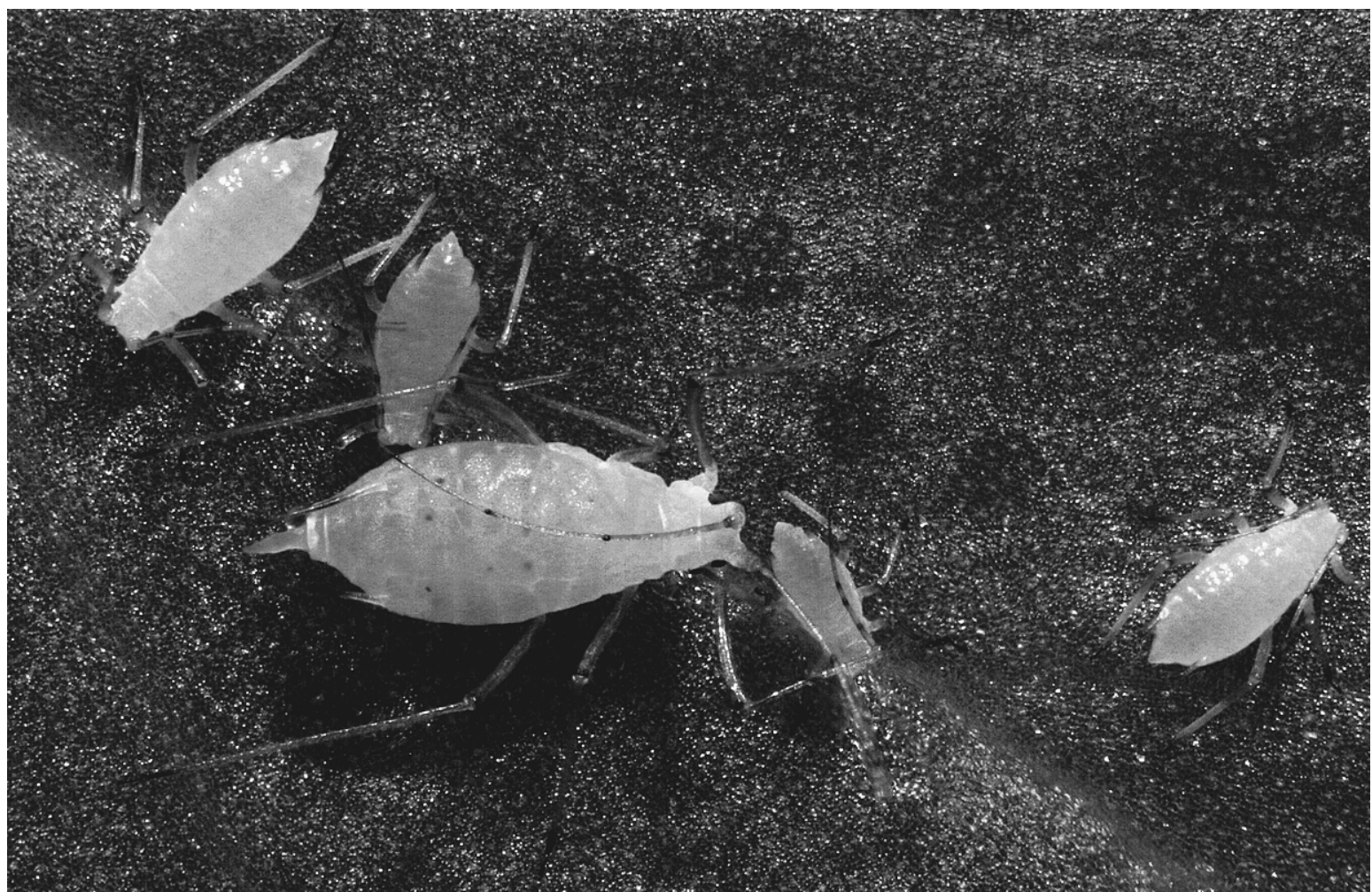

Plate 1. Pea aphids (Acyrthosiphon pisum) on a broad bean leaf. Three different juvenile instars and one adult are visible; eyespots of developing offspring are visible inside the abdomen on the adult. Photo credit: D. Tyre (using a Leica Zoom Stereomicroscope and Helicon Focus to combine multiple images for improved depth of focus).

transient population growth rate may enhance the probability of population establishment. So far transient dynamics have largely been ignored in models predicting population spread (e.g., Kot et al. 1996, Neubert and Caswell 2000, Snyder 2003).

We know of only one other study (Clutton-Brock and Coulson 2002) demonstrating transient dynamics in real populations. How well the predicted transient dynamics match empirical observations depends critically on the number of stages included in the model. In the case of $A$. pisum, the best model incorporated a negative binomial distribution of juvenile stages and a number of adult age classes that sufficiently represented age-dependent fecundity and survival (senescence). The effect of the number of adult stages on transient dynamics is driven by offspring production. Peak aphid reproduction occurred shortly after entering the reproductive stage; six days later offspring production was already reduced by nearly one-half. Virtually all aphids produced on the first day after model initialization went through the juvenile and pre-reproductive stages (low juvenile mortality) in 10 days and entered their most fecund period. The initial large offspring production of the new reproductive adults produced a sharp increase in the population growth rate (Figs. 3 and 4); each following day adults produced a smaller number of offspring, causing population growth rate to decline again. This mechanism is lost when ignoring senescence by combining all adults in a single age class. We have shown elsewhere that incorporating senescence into models can also be critical when predicting long-term dynamics (Lubben et al. 2008).

Other researchers have pointed out that stagespecificity in the vital rates (e.g., senescence in fecundity) influences transient dynamics (Koons et al. 2005, Ramula and Lehtila 2005), but we find that, at least in A. pisum, the number of juvenile stages with comparable vital rates also influenced predicted transient dynamics. Including pseudo-stages in the model essentially adds stages with identical vital rates, and this increased the predicted peak transient population growth rate even further. Including more juvenile stages delays development and ensures that larvae remain in each stage for a minimum period of time. For instance, in the model with two juvenile stages the daily transition rate from the first to the second juvenile stage was 0.26 and from the second juvenile stage to the adult stage was 0.22 (data not shown). Thus, the model predicts that $6 \%$ of aphids $(0.26 \times 0.22)$ reached adulthood in three days, some larger proportion in four days, etc.

Because the model failed to secure a minimum length of the pre-reproductive period of eight days, the frequency distribution of newborns of the second generation is too wide and flat; this reduced the predicted transient peaks. 
This might be a problem in many structured populations, not just aphids. One possible solution is to delay the progress through each larval instar by using a negative binomial distribution of juvenile stages by dividing each juvenile period into $k$ pseudo-stages (Caswell 2001). Yet models incorporating a delay in larval development were not sufficient to correctly predict observed transient population growth; the model using pseudo-stages under-predicted peak transients and hence population size after 20 days (Fig. 6).

Another possibility is that the discrete time step of a matrix projection model (one day in this case) limits the ability of the model to accurately reproduce transient dynamics. However, the discrepancy does not disappear when using a continuous time model with the correct stage structure (partial differential equation [PDE] models); the predictions of a continuous time model are consistent with the matrix using pseudo-stages (B. Tenhumberg and A. J. Tyre, unpublished data).

The perturbation analysis illustrates that a combination of shifting reproduction to earlier ages, decreasing larval development rates, and decreasing larval survival produces transient dynamics that approximately match the experimental time series data. This perturbation increases the predicted asymptotic population growth rate and transient amplification. The perturbation analysis suggests that the inconsistency between observation and model prediction may be the result of biased parameter estimates, which could arise in several different, not mutually exclusive, ways.

First, the temperature during the parameter estimation experiments was on average $2^{\circ} \mathrm{C}$ lower than during the experiment recording transient dynamics. Higher temperature speeds up aphid development and shifts offspring production to younger ages (i.e., young adults have more offspring and old adults have fewer; Morgan et al. 2001). Both factors would result in an increased population growth rate, and higher fecundity increases transient peaks. However, very high temperatures are detrimental to $A$. pisum; reported optimal temperatures vary between $23^{\circ} \mathrm{C}$ (Morgan et al. 2001) and $30^{\circ} \mathrm{C}$ (Siddiqui et al. 1973).

Second, for some aphid species it is beneficial to feed in small groups (Allee effect; Way and Banks 1967, Dixon and Wratten 1971), so estimating model parameters from solitary aphids might underestimate developmental rate, survival, and fecundity. This effect may not be ubiquitous, as other work found no effect of group size on aphid performance (Messina 1993, Hodgson and Godfray 1999). A higher fecundity would produce higher transient peaks (Fig. 3) and consequently higher population size after 20 days. A potential effect on survival is negligible because in our experiments larval mortality was extremely low (only $7.6 \%$ of individuals died before reaching adulthood), thus it was unlikely that the rearing conditions affected larval survival significantly. Additionally, model predictions were insensitive to changes in adult survival (Appendix C).
Third, because aphid fecundity is highly variable between individuals (Fig. 2), it is possible that by chance the founder aphids had above-average fecundity. We distinguished adult aphids by size, which might have biased our choice of founder aphids toward larger adults; adult size and fecundity are positively correlated in many insect species. To avoid a size bias would involve determining the size or mass of live aphids, which would be extremely difficult and might negatively affect aphid's physical condition (e.g., anesthetizing aphids, food deprivation, etc.).

When using our laboratory experiments to parameterize a $3 \times 3$ matrix model, all predicted transient oscillations of our model disappear and the model reaches asymptotic growth very quickly. In contrast, when using the parameters estimated by Gross et al. (2002) the model predicts large oscillations at a higher frequency than our observed populations. This discrepancy is unlikely due to environmental conditions or to the fact that we used laboratory populations and Gross et al. (2002) used field populations, because the model predictions for the three different field populations in Wisconsin (Arlington 1980, 1982, Madison 1980; $\lambda_{\max }=$ $1.35,1.18,1.42$, respectively) differ only in amplitude of the transient peaks and not frequency. There is nothing wrong with the parameter estimation method used by Gross et al. (2002); it is an excellent method to use when only time series data are available. However it is important to fit models to data that include a sufficient amount of complexity to summarize the biology.

It is difficult to visually distinguish between the four larval instars or identify adult age. This is probably why the empirical data used by Gross et al. (2002) only discriminated between small (first and second instar) and large larvae (third and fourth) and adults. But fitting a model with too few stages resulted in somewhat "unrealistic" model parameter estimates. For instance, within a single day $>40 \%$ of aphids move from first- and second-instar stages to the third- and fourth-instar stages and as a consequence the predicted generation times of models by Gross et al. (2002) are 3.1-3.48 days. This is much lower than the generation time recorded for A. pisum populations in Wisconsin (Arlington, 1982, 18.7-11.9 days; Hutchinson and Hogg 1984). In our laboratory experiments, the first aphid that molted to the third instar was four days old and only one out of 66 aphids reached adulthood seven days after birth. In other studies generation time of $\mathrm{A}$. pisum at $20^{\circ} \mathrm{C}$ varied between 6.4 and 9.9 days (Siddiqui et al. 1973, Campbell and Mackauer 1975, Bieri et al. 1983, Lamb 1992, Morgan et al. 2001). Even so, the asymptotic growth rates from the Gross et al. (2002) model are a close match to our empirically observed data.

\section{Conclusion}

In this paper we explored the population dynamics following a dispersal event. We demonstrated that transient dynamics occur after the arrival of a single $A$. 
pisum and that in the absence of density dependence this transient amplification results in considerably higher population sizes. We suspect that transient dynamics may be important for the dispersal dynamics of other species as well, and if this is so, transient dynamics should be considered in theoretical and empirical dispersal studies. This paper also demonstrates that the number of lifehistory stages is critical for correctly predicting transient dynamics; this suggests that structural uncertainty should not be ignored in model development. Furthermore, this paper points to some challenges involved with testing model predictions empirically. Biases in parameter estimates as a result of small variations in temperature or rearing conditions were likely responsible for the large discrepancy in predicted and observed transient dynamics, with the consequence of underestimating transient amplification $\left(T_{20}=4.29\right.$ instead of 3.06). If the variation in vital rates of field populations as a result of temporal and spatial variation is similar or larger than in our study we have to treat the quantitative estimates from population viability analysis based on density-independent matrix models with caution.

\section{ACKNOWLEDGMENTS}

We are very thankful to John Reese (Kansas State University) who kindly provided us with advice on rearing $A$. pisum in the laboratory. The experimental work greatly benefited from the dedication of Masaru Takahashi, who spent endless hours counting thousands of aphids. Comments by David N. Koons, Matthew Spencer, Tom E. X. Miller, Travis M. Hinkelman, and two anonymous reviewers greatly improved our manuscript.

\section{Literature Cited}

Allen, L. J. S., J. F. Fagan, G. Hognas, and H. Fagerholm. 2005. Population extinction in discrete-time stochastic population models with an Allee effect. Journal of Difference Equations and Applications 11:273-293.

Berggren, A. 2001. Colonization success in Roesel's bushcricket Metrioptera roeseli: the effects of propagule size. Ecology 82:274-280.

Bieri, M., J. Baumgartner, G. Bianchi, V. Delucchi, and R. von Arx. 1983. Development and fecundity of pea aphid (Acyrthosiphon pisum Harris) as affected by constant temperature and by pea cultivars. Bulletin de la Société Entomologique Suisse 56:163-171.

Bierzychudek, P. 1999. Looking backwards: assessing the projections of a transition matrix model. Ecological Applications 9:1278-1287.

Burgman, M. A., S. Ferson, and H. R. Akçakaya. 1993. Risk assessment in conservation biology. Chapman and Hall, New York, New York, USA.

Campbell, A., and M. Mackauer. 1975. Thermal constants for development of the pea aphid (Homoptera: Aphididae) and some of its parasites. Canadian Entomologist 107:419-423.

Campbell, A., and M. Mackauer. 1977. Reproduction and population growth of the pea aphid (Homoptera: Aphididae) under laboratory and field conditions. Canadian Entomologist 109:277-284.

Caswell, H. 2001. Matrix population models. Second edition. Sinauer, Sunderland, Massachusetts, USA.

Caswell, H. 2007. Sensitivity analysis of transient population dynamics. Ecology Letters 10:1-15.

Caswell, H., and M. G. Neubert. 2005. Reactivity and transient dynamics of discrete-time ecological systems. Journal of Difference Equations and Applications 11:295-310.
Clutton-Brock, T. H., and T. Coulson. 2002. Comparative ungulate dynamics: the devil is in the detail. Philosophical Transactions of the Royal Society B 357:1285-1298.

Cox, D. R., and D. Oakes. 1984. Analysis of survival data. Chapman and Hall, London, UK.

Dixon, A. 1985. Aphid ecology. Chapman and Hall, London, UK.

Dixon, A. F. G., and S. D. Wratten. 1971. Laboratory studies on aggregation, size and fecundity in the black bean aphid, Aphis fabae Scop. Bulletin of Entomological Research 61:97111.

Drake, J. M. 2005. Risk analysis for invasive species and emerging infectious diseases: concepts and applications. American Midland Naturalist 153:4-19.

Efron, B., and R. J. Tibshirani. 1993. An introduction to the bootstrap. Chapman and Hall, New York, New York, USA. Fagan, W. F., M. Lewis, M. G. Neubert, C. Aumann, J. L. Apple, and J. G. Bishop. 2005. When can herbivores slow or reverse the spread of an invading plant? A test case from Mount St. Helens. American Naturalist 166:669-685.

Gotelli, N. 2001. A primer of ecology. Third edition. Sinauer, Sunderland, Massachusetts, USA.

Grevstad, F. S. 1999. Factors influencing the chance of population establishment: implications for release strategies in biocontrol. Ecological Applications 9:1439-1447.

Grimm, V., E. Revilla, U. Berger, F. Jeltsch, W. M. Mooij, S. F. Railsback, H. H. Thulke, J. Weiner, T. Wiegand, and D. L. DeAngelis. 2005. Pattern-oriented modeling of agentbased complex systems: lessons from ecology. Science 310: 987-991.

Gross, K., B. A. Craig, and W. D. Hutchinson. 2002. Bayesian estimation of a demographic matrix model from stagefrequency data. Ecology 83:3285-3298.

Hastings, A. 2004. Transients: the key to long-term ecological understanding? Trends in Ecology and Evolution 19:39-45.

Hodgson, D. J., and H. C. J. Godfray. 1999. The consequences of clustering by Aphis fabae foundresses to spring migrant production. Oecologia 118:446-452.

Hodgson, D. J., and S. Townley. 2004. Linking management changes to population dynamic responses: the transfer function of a projection matrix perturbation. Journal of Applied Ecology 41:1155-1161.

Hutchinson, W. D., and D. B. Hogg. 1984. Demographic statistics for the pea aphid (Homoptera: Aphididae) in Wisconsin and a comparison with other populations. Environmental Entomology 13:1173-1181.

Hutchinson, W. D., and D. B. Hogg. 1985. Time-specific life tables for the pea aphid, Acyrthosiphon pisum (Harris), on alfalfa. Researches on Population Ecology 27:231-253.

Keitt, T. H., M. A. Lewis, and R. D. Holt. 2001. Allee effects, invasion pinning, and species' borders. American Naturalist 157:203-216.

Koons, D. N., J. B. Grand, B. Zinner, and R. E. Rockwell. 2005. Transient population dynamics: relations to life history and initial population state. Ecological Modelling 185:283297.

Koons, D. N., R. R. Holmes, and J. B. Grand. 2007. Population inertia and its sensitivity to changes in vital rates and population structure. Ecology 88:2857-2867.

Kot, M., M. A. Lewis, and P. vandenDriessche. 1996. Dispersal data and the spread of invading organisms. Ecology 77:20272042.

Lamb, R. J. 1992. Developmental rates of Acyrthosiphon pisum (Homoptera: Aphididae) at low temperatures: implications for estimating rate parameters for insects. Environmental Entomology 21:10-19.

Lubben, J., B. Tenhumberg, A. J. Tyre, and R. Rebarber. 2008. Management recommendations based on matrix projection models: the importance of considering biological limits. Biological Conservation 14:517-523.

McCallum, H., editor. 2000. Population parameters. Blackwell Science, London, UK. 
Messina, F. J. 1993. Effect of initial colony size on the percapita growth-rate and alate production of the Russian wheat aphid (Homoptera, Aphididae). Journal of the Kansas Entomological Society 66:365-371.

Morgan, D., K. F. Walters, and J. N. Aegerter. 2001. Effect of temperature and cultivar on pea aphid, Acyrthosiphon pisum (Hemiptera: Aphididae) life history. Bulletin of Entomological Research 91:47-52.

Morris, W. F., and D. F. Doak. 2002. Quantitative conservation biology: theory and practice in conservation biology. Sinauer, Sunderland, Massachusetts, USA

Nelson, E. H. 2007. Predator avoidance behavior in the pea aphid: costs, frequency, and population consequences. Oecologia 151:22-32.

Nelson, E. H., and J. A. Rosenheim. 2006. Encounters between aphids and their predators: the relative frequencies of disturbance and consumption. Entomologia Experimentalis et Applicata 118:211-219.

Neubert, M. G., and H. Caswell. 1997. Alternatives to resilience for measuring the responses of ecological systems to perturbations. Ecology 78:653-665.

Neubert, M. G., and H. Caswell. 2000. Demography and dispersal: calculation and sensitivity analysis of invasion speed for structured populations. Ecology 81:1613-1628.

Pfister, C. A., and F. R. Stevens. 2003. Individual variation and environmental stochasticity: implications for matrix model predictions. Ecology 84:496-510.

R Development Core Team. 2006. R: a language and environment for statistical computing. R Foundation for Statistical Computing, Vienna, Austria. 〈http://www.r-project.org

Ramula, S., and K. Lehtila. 2005. Matrix dimensionality in demographic analyses of plants: When to use smaller matrices? Oikos 111:563-573.

Shea, K., and H. P. Possingham. 2000. Optimal release strategies for biological control agents: an application of stochastic dynamic programming to population management. Journal of Applied Ecology 37:77-86.
Siddiqui, W. H., C. A. Barlow, and P. A. Randolph. 1973. Effects of some constant and alternating temperatures on population growth of the pea aphid, Acyrthosiphon pisum (Homoptera: Aphididae). Canadian Entomologist 105:145-156.

Snyder, R. E. 2003. How demographic stochasticity can slow biological invasions. Ecology 84:1333-1339.

Stiling, P. 1990. Calculating the establishment rates of parasitoids in classical biological control. American Entomologist 36:225-282.

Taylor, C. M., and A. Hastings. 2005. Allee effects in biological invasions. Ecology Letters 8:895-908.

Tenhumberg, B. 2004. Predicting predation efficiency of biocontrol agents: linking behavior of individuals and population dynamics. Pages 864-869 in C. Pahl, S. Schmidt, and T. Jakeman, editors. Complexity and integrated resources management: transactions of the 2nd Biennial Meeting of the International Environmental Modelling and Software Society. Manno, Lugano, Switzerland.

Tenhumberg, B., A. J. Tyre, A. R. Pople, and H. P. Possingham. 2004a. Do harvest refuges buffer kangaroos against evolutionary responses to selective harvesting? Ecology 85:2003-2017.

Tenhumberg, B., A. J. Tyre, K. Shea, and H. P. Possingham. $2004 b$. Linking wild and captive population to maximize species persistence: optimal translocation strategies. Conservation Biology 18:1304-1314.

Townley, S., D. Carslake, O. Kellie-Smith, D. McCarthy, and D. Hodgson. 2007. Predicting transient amplification in perturbed ecological systems. Journal of Applied Ecology 44: $1243-1251$.

Tyre, A. J., D. G. Kerr, B. Tenhumberg, and C. M. Bull. 2007. Identifying mechanistic models of spatial behaviour using pattern-based modelling: an example from lizard home ranges. Ecological Modelling 208:307-316.

Way, M. J., and C. J. Banks. 1967. Intra-specific mechanisms in relation to the natural regulation of numbers of Aphis fabae Scop. Annales of Applied Biology 59:189-205.

\section{APPENDIX A}

Matrix model of Acyrthosiphon pisum (Ecological Archives E090-130-A1).

\section{APPENDIX B}

Effect of initial conditions on transient amplification after 20 days (Ecological Archives E090-130-A2).

\section{APPENDIX C}

Perturbation analysis of the Acyrthosiphon pisum model that includes larval pseudo-stages (Ecological Archives E090-130-A3).

\section{APPENDIX D}

Transient amplification $\left(T_{20}\right)$ as a function of asymptotic population growth rate $(\lambda)$ (Ecological Archives E090-130-A4). 
Brigitte Tenhumberg, Andrew J. Tyre, and Richard Rebarber. 2009. Model complexity affects transient population dynamics following a dispersal event: a case study with pea aphids. Ecology 90:1878-1890.

Appendix A. Matrix model of Acyrthosiphon pisum.

$\mathrm{L}_{1}-\mathrm{L}_{4}$ indicate 1 st -4 th instar, $\mathrm{A}_{1}$ is the pre-reproductive, and $\mathrm{A}_{2}-\mathrm{A}_{7}$ are reproductive adult stages. $\mathrm{A}_{1}$ is greater than zero because some pre-reproductive adults graduate to the reproductive stage during the census interval.

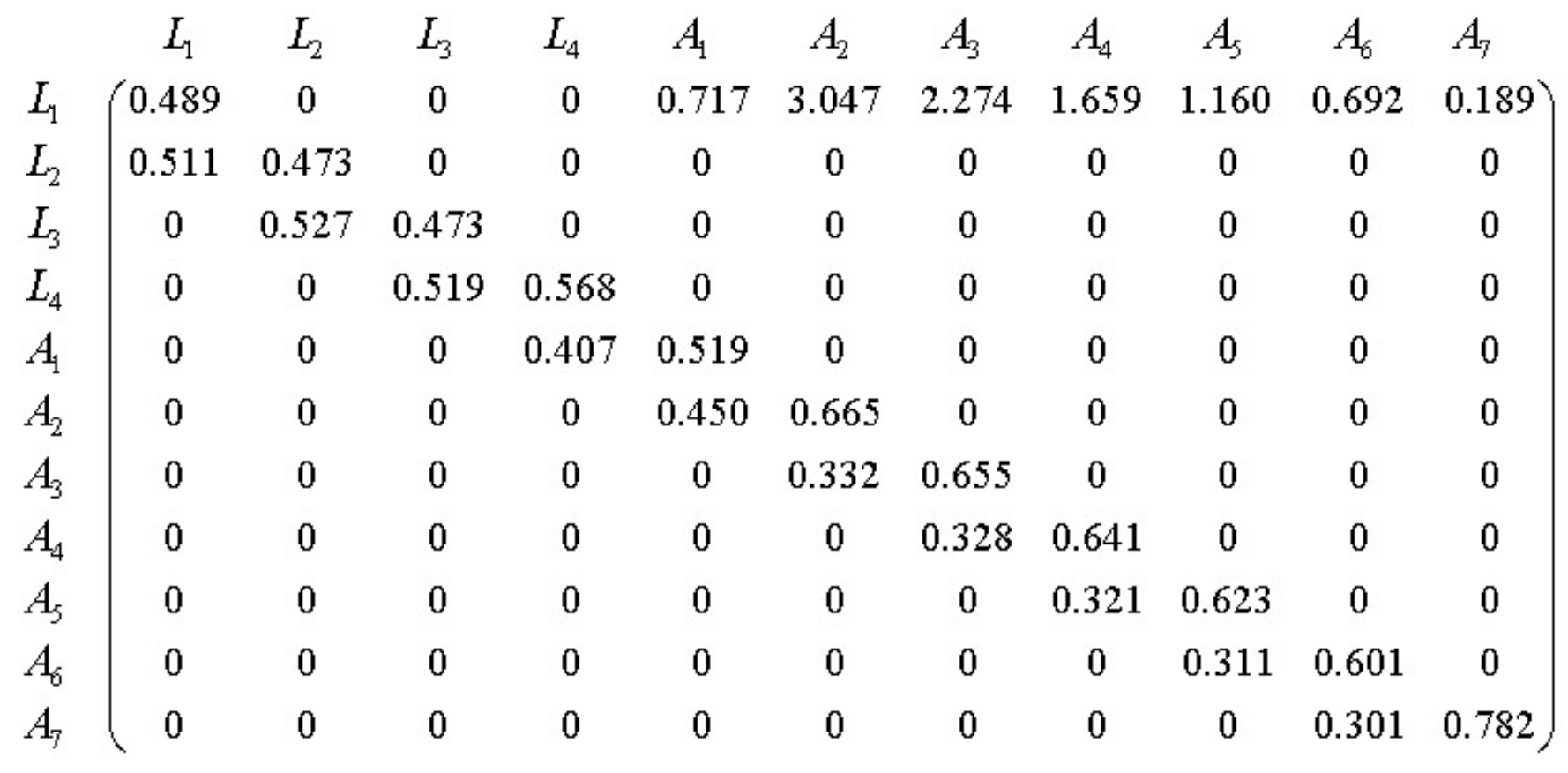




\section{Ecological Archives E090-130-A2}

Brigitte Tenhumberg, Andrew J. Tyre, and Richard Rebarber. 2009. Model complexity affects transient population dynamics following a dispersal event: a case study with pea aphids. Ecology 90:1878-1890.

Appendix B. Effect of initial conditions on transient amplification, TR, after 20 days.

$T R=\left(\sum_{i} n_{i, 20}\right) /\left(\lambda_{\max }\right)^{19}$, where $n_{i, t}$ indicates the number of aphids in stage class $i$ on day $t$ 


\begin{tabular}{|c|c|c|}
\hline 2 jure rile stages +2 adult stages & $(0,0,1,0)$ & 1.92 \\
\hline$\lambda_{\max }=1.234$ & $(0,0,0,1)$ & 2.64 \\
\hline 4 juve nile stages +2 adult stages & $(0,0,0,0,1,0)$ & 1.95 \\
\hline$\lambda_{\max }=1.212$ & $(0,0,0,0,0,1)$ & 2.60 \\
\hline 2 juve nile stages +7 adult stages & $(0,0,1,0,0,0,0,0,0)$ & 2.46 \\
\hline \multirow[t]{6}{*}{$\lambda_{\max }=1.302$} & $(0,0,0,1,0,0,0,0,0)$ & 3.53 \\
\hline & $(0,0,0,0,1,0,0,0,0)$ & 2.49 \\
\hline & $(0,0,0,0,0,1,0,0,0)$ & 1.68 \\
\hline & $(0,0,0,0,0,0,1,0,0)$ & 1.04 \\
\hline & $(0,0,0,0,0,0,0,1,0)$ & 0.53 \\
\hline & $(0,0,0,0,0,0,0,0,1)$ & 0.17 \\
\hline 4 jwvenile stages +7 adult stage & $(0,0,0,0,1,0,0,0,0,0,0)$ & 2.49 \\
\hline \multirow[t]{6}{*}{$\lambda_{\max }=1.265$} & $(0,0,0,0,0,1,0,0,0,0,0)$ & 3.42 \\
\hline & $(0,0,0,0,0,0,1,0,0,0,0)$ & 2.40 \\
\hline & $(0,0,0,0,0,0,0,1,0,0,0)$ & 1.60 \\
\hline & $(0,0,0,0,0,0,0,0,1,0,0)$ & 0.98 \\
\hline & $(0,0,0,0,0,0,0,0,0,1,0)$ & 0.50 \\
\hline & $(0,0,0,0,0,0,0,0,0,0,1)$ & 0.16 \\
\hline 7 juvenile pee ub-stages +7 adult & $(0,0,0,0,0,0,0,1,0,0,0,0,0,0)$ & 2.74 \\
\hline stages & $(0,0,0,0,0,0,0,0,1,0,0,0,0,0)$ & 3.06 \\
\hline \multirow[t]{5}{*}{$\lambda_{\max }=1.280$} & $(0,0,0,0,0,0,0,0,0,1,0,0,0,0)$ & 2.14 \\
\hline & $(0,0,0,0,0,0,0,0,0,0,1,0,0,0)$ & 1.41 \\
\hline & $(0,0,0,0,0,0,0,0,0,0,0,1,0,0)$ & 0.85 \\
\hline & $(0,0,0,0,0,0,0,0,0,0,0,0,1,0)$ & 0.43 \\
\hline & $(0,0,0,0,0,0,0,0,0,0,0,0,0,1)$ & 0.14 \\
\hline
\end{tabular}


Brigitte Tenhumberg, Andrew J. Tyre, and Richard Rebarber. 2009. Model complexity affects transient population dynamics following a dispersal event: a case study with pea aphids. Ecology 90:1878-1890.

Appendix C. Perturbation analysis of the Acythosiphon pisum model that includes larval pseudo-stages.

\section{Adult Survival}

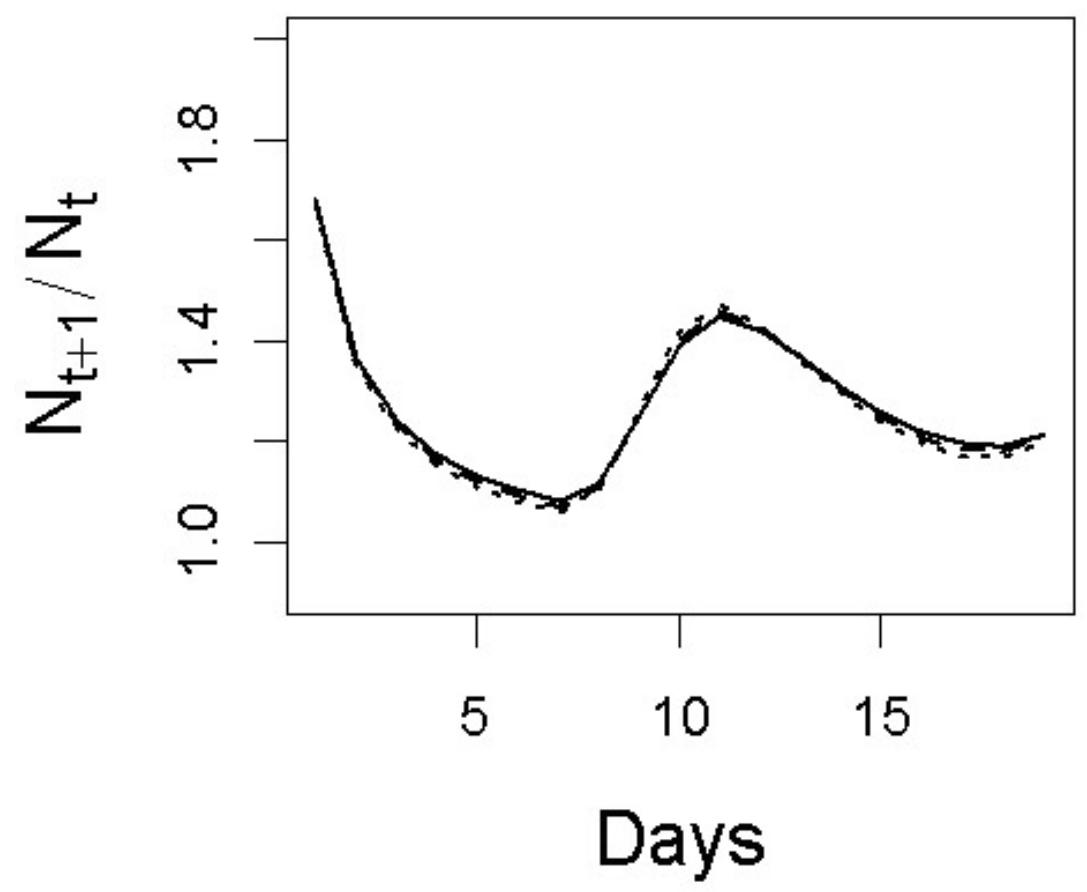

FIG. C1. Default values are listed in Table 3 and the resulting predictions are indicated by solid curves. Adult survival: With increasing $\rho$ survival decreases faster; solid curve: $\rho=0.058025$, dashed curve: $\rho=0.08$, dotted curve: $\rho=0.1$. 


\section{Ecological Archives E090-130-A4}

Brigitte Tenhumberg, Andrew J. Tyre, and Richard Rebarber. 2009. Model complexity affects transient population dynamics following a dispersal event: a case study with pea aphids. Ecology 90:1878-1890.

Appendix D. Transient amplification $\left(T_{20}\right)$ as a function of asymptotic population growth rate $(\lambda)$.

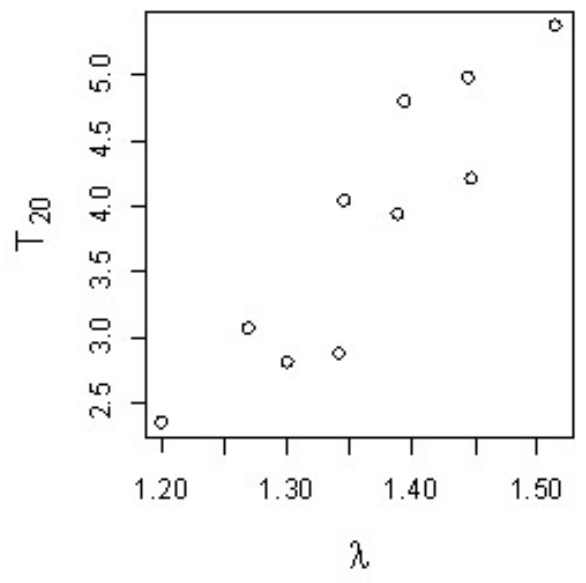

FIG. D1. Calculations are based on parameter estimated of Fig. 7; to include a smaller $\lambda$-value we also calculated $\lambda$ and $T_{20}$ assuming fecundity is half the unperturbed value $\left(T_{20}=-9.77+10.05 \lambda, r^{2}=0.78, P<0.001\right)$. 